\title{
Influence of the surface charge on the homogeneity of colloidal crystals
}

\author{
Hongwei Zhou, ${ }^{1,2}$ Shenghua Xu, ${ }^{1,2, a)}$ Wenze Ouyang, ${ }^{1,2}$ Zhiwei Sun, ${ }^{1,2, a)}$ and Lixia Liu ${ }^{1,2}$ \\ ${ }^{1}$ Key Laboratory of Microgravity, Institute of Mechanics, Chinese Academy of Sciences, \\ No. 15 Beisihuanxi Road, Beijing 100190, People's Republic of China \\ ${ }^{2}$ National Microgravity Laboratory, Institute of Mechanics, Chinese Academy of Sciences, \\ No. 15 Beisihuanxi Road, Beijing 100190, People's Republic of China
}

(Received 2 April 2013; accepted 2 July 2013; published online 12 August 2013)

\begin{abstract}
Five groups of suspensions composed of polystyrene particles, having similar size but different effective surface charge, were adopted to investigate the effects of surface charge and volume fraction on the homogeneity of colloidal crystals through checking the difference between $D_{\text {exp }}$ and $D_{\text {uni }}$ by reflection spectroscopy method $\left(D_{\text {exp }}, D_{\text {uni }}\right.$ are the experimental and the expected value of the average nearest neighbor interparticle distance by assuming a uniform structure, respectively). We found volume fractions (ranging from 0.006 to 0.02 ) and structure types basically have no influence on the values of $D_{\text {exp }} / D_{\text {uni }}$. Moreover, for crystals formed by lowly charged particles, $D_{\text {exp }} / D_{\text {uni }}$ is approximately equal to 1 , implying the crystals are homogeneous. With the increase of effective surface charge, $D_{\text {exp }}$ gradually deviates from $D_{u n i}$ and the formed crystals become inhomogeneous. Our experimental observations are in accordance with the previous simulation results. Additionally, we also found $D_{\text {exp }} / D_{\text {uni }}$ initially drops quickly with increasing effective surface charge and then it tends to an asymptotic value $(\sim 0.85)$, it is supposedly due to the saturation of effective charge. Our relevant computer simulations confirmed that the study scheme that using $D_{\text {exp }} / D_{\text {uni }}$ as an indicator to assess the homogeneity of crystal structure is tenable and the simulation results are consistent with experiments. (C) 2013 AIP Publishing LLC. [http://dx.doi.org/10.1063/1.4815920]
\end{abstract}

\section{INTRODUCTION}

Charged colloidal particles suspended in de-ionized water under appropriate conditions can be self-assembled into highly ordered arrays of particles: colloidal crystals. ${ }^{1,2}$ This self-assembly process of colloids directly appears analogous to their atomic or molecular counterparts with proper scaling considerations and therefore can offer a useful model system for studying general principles of the crystallization of materials. ${ }^{3}$ Since time and length scales in colloidal crystals are several orders of magnitude larger than those of atomic or molecular crystals, one can acquire various more suitable tools and instruments for observation and measurement in investigation of crystallization to gain an insight into crystal growth., ${ }^{4,5}$

It has been generally acknowledged that the crystalline structure of like-charged particles is formed due to the pure repulsive interparticle interaction that can be described by the DLVO (Derjaguin-Landau-Verwey-Overbeek) theory, which combines the effects of the van der Waals attraction (shortranged and negligibly small for interparticle distance exceeds $200 \mathrm{~nm})^{6}$ and the electrostatic repulsion. In practice, Yukawa potential, based on DLVO theory and referred to as the screened Coulomb repulsion, has been widely used in modeling various substances in category of soft matter. ${ }^{7,8}$ However, there are some phenomena ${ }^{9-13}$ that may not be explained rationally within the framework of the DLVO model.

\footnotetext{
a) Authors to whom correspondence should be addressed. Electronic addresses: xush@imech.ac.cn and sunzw@imech.ac.cn. Tel.: +86 10 82544099. Fax: +86 1082544096.
}

Among these "puzzling phenomena," a straightforward example is the structural inhomogeneity (namely, the crystal region contains some voids) of colloidal crystal in chargestabilized colloidal suspensions. The argument is that if only repulsions exist between charged particles, the ordered structures should be uniformly distributed in the container without voids. However, some experimental observations indicated that there are voids, ${ }^{14,15}$ as well as some "self-sustaining structures" (localized, ordered structures of particles) ${ }^{16}$ appeared in charged colloidal crystals. This structural inhomogeneity in crystal directly challenges the repulsion-only Yukawa potential (the short-ranged attractions are completely masked by Coulomb repulsions). To explain this structural inhomogeneity, inclusion of a long-range attraction, in addition to repulsion interaction, between particles has been suggested. ${ }^{12,17}$ More specifically, the Sogami-Ise (SI) potential $^{18}$ was proposed in order to overcome the abovementioned difficulty of the Yukawa potential.

Until now, however, whether a long-range attraction really exists still remains a quite controversial issue, see the related papers by Overbeek, ${ }^{19}$ van Roij, ${ }^{20,21}$ Schmitz, ${ }^{22,23}$ Smalley, ${ }^{24}$ and controversies between Croker and Tata, ${ }^{25}$ Mulder and Ise, ${ }^{26}$ etc.

Apparently, more extensive study on the paradox of the repulsion-only assumption in different ways is necessary. As far as we know, for lowly charged particles, there are rare problems reported in literatures on the applicability of DLVO theory, indicating the repulsion-only assumption is a good approximation. The riddles seem to mostly happen to crystal formed by highly charged particles. This situation motivated us to study how the actual charge levels of particles affect the 
structural homogeneity of the colloidal crystals. Another parameter that may influence the structural homogeneity is volume fraction. This paper is aimed at exploring whether and how particle charge and volume fraction affect the structural homogeneity.

If voids exist, the average nearest neighbor inter-particle distance $(A N N I D)$ would be, due to a reduction in overall volume of the ordered region, less than that when the ordered structures is uniformly distributed. Therefore, the magnitude of ANNID for a specific colloidal crystal system has previously been used ${ }^{12,16}$ to judge whether its structure is homogeneous (ordered colloidal particles occupy the entire volume of the container) or not.

Let $D_{\text {uni }}$ be $A N N I D$ for the uniform structure and $D_{\text {exp }}$ be that obtained from actual experiments. In the present study, we take the ratio of $D_{\text {exp }}$ to $D_{\text {uni }}$ (namely, $D_{\text {exp }} / D_{\text {uni }}$ ) to be an indicator of the degree of structural homogeneity in crystal. For uniform structure, $D_{\text {exp }} / D_{\text {uni }}$ takes its maximum value, 1.0. If the structure is inhomogeneous, $D_{\text {exp }}<D_{u n i}$ and therefore $D_{\text {exp }} / D_{\text {uni }}<1 .{ }^{12}$

Our experimental results in this study show how $D_{\text {exp }} / D_{\text {uni }}$ is related to effective surface charge $Z_{e}$ and volume fraction $\Phi$. Five groups of suspensions composed of polystyrene (PS) particles having similar size $(\sim 101 \mathrm{~nm})$ but different $Z_{e}$ was adopted to reveal the relationship between $D_{\text {exp }} / D_{\text {uni }}$ and $Z_{e}$, and in each group eight subcases with different $\Phi$ were used to investigate the $\Phi$-dependence of $D_{\text {exp }} / D_{\text {uni }}$, by means of reflection spectroscopy (RS) method. We found that $D_{\text {exp }} / D_{\text {uni }}$ is insensitive to the change of $\Phi$ within the range where the experiments were performed, but it decreases with increasing $Z_{e}$ until an asymptotic value is reached. In addition, our computer simulations using SI potential seem to be consistent with the experimental results.

The rest of the paper is organized as follows. Section II describes the experimental method and computer simulation framework. Section III provides our results and discussions. Finally, conclusions are given in Sec. IV.

\section{EXPERIMENTAL METHOD AND COMPUTER SIMULATION}

\section{A. Preparation and characterization of PS particles}

The PS particles used in the experiments were synthesized by an emulsion polymerization method. ${ }^{27}$ The prepared PS particles carrying a large number of ionizable sulfate groups, which are directly related to bare charge of particles. By varying the amount of $\mathrm{K}_{2} \mathrm{~S}_{2} \mathrm{O}_{8}$ in the synthesis, the grafted surface groups can be controlled, yielding particles with different bare charge, represented by analytical charge $Z_{a}$, which can be determined by conductometric titration. Due to limited skill and expertise, we cannot ensure so-produced particles to be quite identical in size and also to have desired surface charge. Prepared PS particles were stored with resin (G501X8(D), Bio-Rad Laboratories, USA) for further deionization.

The diameter and polydispersity of the particles were measured by means of dynamic light scattering (DLS, Brookhaven Instruments Corp., USA) and the values were also verified by transmission electron microscopy (TEM, JEM-2100).

\section{B. Effective charge determination}

In this study, the particle radius is so much smaller than the interparticle distance due to strong repulsion that charge numbers become a dominant parameter. When PS colloidal particles are suspended in a polar solvent (usually water), the sulfate groups dissociate, leaving negative charge at the surface and counterions (proton) into the solutions. However, due to the accumulation of micro-ions around highly charged colloidal particles in electrolyte solution, the degree of dissociation $f$ is usually much less than unity. ${ }^{28}$ Therefore, the effective charge $Z_{e}$, which is the essential parameter to determine the interactions, can be quite low as compared to analytical charge $Z_{a}$. Hessinger ${ }^{29}$ proposed a conductivity model to estimate the effectively transported charge $Z_{\sigma}^{*}$ quantitatively. In the case of deionized suspensions, assuming the additivity of all conductivity contributions, the conductivity of the suspension is given by

$$
\sigma=\sigma_{0}+\sigma_{B}=n e Z_{\sigma}^{*}\left(\mu_{p}+\mu_{H^{+}}\right)+\sigma_{B},
$$

where $\sigma_{0}$ is the conductivity of particles plus counterions and $\sigma_{B}$ the background conductivity, $n$ is the particle number density and $e$ elementary charge, $\mu_{H^{+}}$and $\mu_{p}$ is the mobility of protons and charged particles, respectively. $\mu_{H^{+}}$is 36.5 $\times 10^{-8} \mathrm{~m}^{2} \cdot \mathrm{V}^{-1} \cdot \mathrm{s}^{-1}$. The plateau value of $\mu_{p}$ is chosen as in Refs. 29 and 30 because it saturates at a large value of $n$. Thus, the effectively transported charge $Z_{\sigma}^{*}$ is taken as the only free fitting parameter in the $\sigma-n$ relationship of Eq. (1). A typical $\sigma-n$ relationship for particles PS1 is shown in Fig. 1. In the range of $n$ concerned in this study, $\sigma$ and $n$ keeps strictly linear, in despite of the first order freezing transition $n \approx 3.2 \mu \mathrm{m}^{-3}(\Phi=0.002)$. One parameter linear fitting of $\sigma-n$ in Fig. 1 yields $Z_{\sigma}^{*}=561$. Similar result is also observed for the other particles and the freezing happens around $n \approx 3-6 \mu \mathrm{m}^{-3}$. $Z_{\sigma}^{*}$ of the rest groups of particles, sorted in

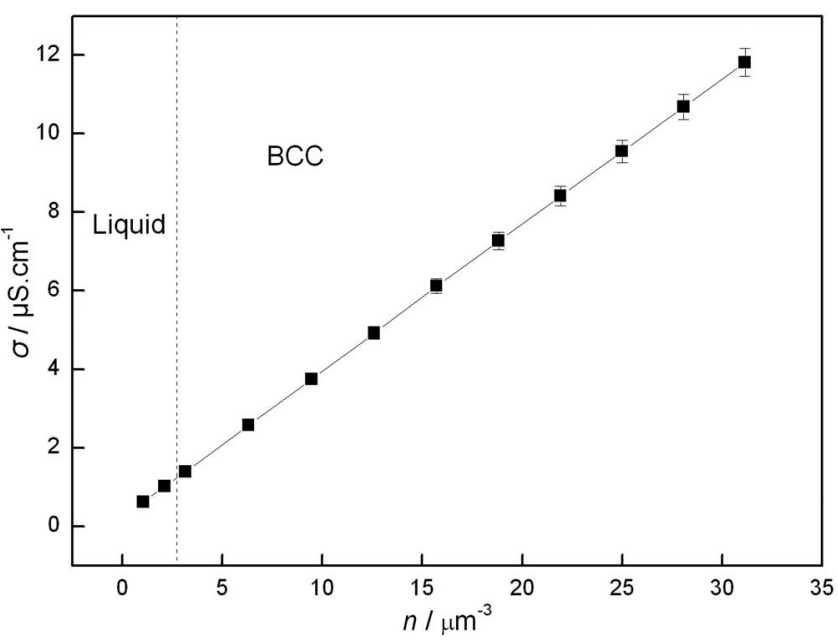

FIG. 1. The conductivity $\sigma$ of the suspension as a function of particle number density $n$ for particles PS1. The vertical dotted line is a dividing line between liquid-like and solid-like (BCC) phase. 
TABLE I. Parameters of the colloidal particles.

\begin{tabular}{lcccc}
\hline \hline Sample & $\begin{array}{c}\text { Radius } \\
(\mathrm{a}, \mathrm{nm})\end{array}$ & Polydispersity & $\begin{array}{c}\text { Analytical } \\
\text { charge }\left(Z_{a}\right)\end{array}$ & $\begin{array}{c}\text { Effective } \\
\text { charge }\left(Z_{\sigma}^{*}\right)^{\mathrm{a}}\end{array}$ \\
\hline PS1 & 53 & 0.04 & 9085 & $561 \pm 29$ \\
PS2 & 48 & 0.05 & 13005 & $721 \pm 32$ \\
PS3 & 52 & 0.03 & 15283 & $770 \pm 36$ \\
PS4 & 58 & 0.04 & 25602 & $998 \pm 28$ \\
PS5 & 51 & 0.03 & 18233 & $1003 \pm 34$ \\
\hline \hline
\end{tabular}

${ }^{a}$ Asorted in increasing order of effective surface charge.

increasing order of effective charge in Table I, were determined similarly.

\section{Sample preparation}

Our experiment is arranged for the $D_{\text {exp }}$ measurement for particles with different $Z_{\sigma}^{*}$ and $\Phi$. Suspensions were divided into five experimental groups depending of $Z_{\sigma}^{*}$ and each group has eight subcases corresponding to eight different $\Phi$ raging from 0.006 to 0.02 . The samples used were prepared by carefully mixing the stock latex with a certain amount of pure water in vials, and then the mixtures were sonicated for several minutes to attain homogeneous ones. After some resin added, the vials were sealed with laboratory film (PARAFILM ${ }^{\circledR} \mathrm{M}$, Chicago) and put on rotation stage to deionization. The particles' volume fraction $\Phi$ (or number density $n$ ) and degree of deionization were checked again after $D_{\text {exp }}$ measurements were completed by conductivity measurements.

\section{Characterization of colloidal crystal}

Our equipment is composed of a fiber optic spectrometer (Avantes, Avaspec-2048, Netherlands) with a tungsten halogen light source (Avalight-HAL, Netherlands) and a bifurcated fiber optic cable. By shaking, the formed crystal can melt and after the shaking stops the process of recrystallization starts. The measurement was performed only after a stable crystal structure was formed, and similar measurements were repeated for several times at different positions of the sample cell to get the average $D_{\text {exp }}$ for each subcase.

Reflection spectroscopy has been employed to determine the crystal structure and $D_{\text {exp }}$ in colloidal crystals. ${ }^{31-34}$ Based on the Bragg's law, we can get $2 d \sin \theta=m \lambda / \nu$, where $d$ is interplanar distance, $\theta$ is Bragg angle, $m$ is integer, and $v$ is refractive index of medium so that $\lambda / \nu$ is the wavelength in medium. In PS colloidal dispersions, $v$ can be estimated from the indices of water and PS and the volume fraction by $v_{\text {sample }}=v_{\text {water }}(1-\phi)+v_{p s} \phi$. For the measurement of interplanar distance by RS with normal incidence as shown in our previous studies, ${ }^{33,34}$ Bragg angle is $90^{\circ}$, then lattice constant $l$ can be written as

$$
l=(m \lambda / 2 \nu) \sqrt{h^{2}+k^{2}+l^{2}} .
$$

$D_{\text {exp }}$ can then be deduced from lattice constant $l$ as follows.

For $\lambda$ corresponding to the (110) plane of bcc-like structure, which can be determined by reflection spectrum as in our previous studies, ${ }^{34} D_{\text {exp }}$ is

$$
\begin{aligned}
D_{\exp } & =\frac{\sqrt{3}}{2} l=\frac{\sqrt{3}}{2} \frac{m \lambda}{2 v} \sqrt{1^{2}+1^{2}+0^{2}} \\
& =\frac{\sqrt{6}}{4 v} m \lambda=0.6124 m \lambda / \nu .
\end{aligned}
$$

For $\lambda$ corresponding to the (111) plane of fcc-like structure:

$$
\begin{aligned}
D_{\exp } & =\frac{\sqrt{2}}{2} l=\frac{\sqrt{2}}{2} \frac{m \lambda}{2 v} \sqrt{1^{2}+1^{2}+1^{2}} \\
& =\frac{\sqrt{6}}{4 v} m \lambda=0.6124 m \lambda / \nu .
\end{aligned}
$$

From the above derivation, we can see that the same formula

$$
D_{\exp }=0.6124 m \lambda / v
$$

is suitable for both bcc and fcc-like structures.

\section{E. Computer simulation}

The study approach by means of ANNID measurement presented in this paper is based on the premise that inclusion of attraction besides repulsion interaction between particles makes the ordered structures to have some voids inside, rather than to homogeneously fill the entire accessible volume. To justify this physical picture, we also performed computer simulations using the molecular dynamics (MD) method.

A typical MD simulation is carried out in the NVT ensemble. The period boundary condition is applied and the equation of particles' motion is integrated using velocity Verlet algorithm. ${ }^{35}$ The constant temperature is controlled via Berendsen thermostat. ${ }^{36}$ Initially, we place the particles $(N=4000)$ randomly in a three-dimensional cubic simulation box (see Fig. 2(a)). After a long enough periods of MD time steps, the system can reach equilibrium so that some parameters needed are calculated. The parameters adopted in the simulations are compatible with those for suspension of PS5 (the corresponding parameters used in simulations can be seen in Table I, and the salt concentration is estimated to be $3.0 \times 10^{-6} \mathrm{M}$ according to the conductivity of water, ${ }^{15}$ volume fraction is 0.01 ). The interparticle pair potential was chosen to be Yukawa (repulsion only) or SI form (with longrange attraction) for comparison.

From the simulations, we can find the following characteristics for the colloidal structures:

1. When only Yukawa potential is used, the formed ordered structures homogenously fill the entire accessible space (see Fig. 2(b)).

2. When the SI potential, which takes the long-range attraction into account, is used, voids appear in the ordered structures. However, in crystal regions the structures are still homogenous to a great extent (see Fig. 2(c)).

3. The ANNID value for case 2 is smaller than that for 1 apparently because of the shrinkages of crystal regions due to the existence of voids.

More detailed data and discussions about the simulations will be given in Sec. III. Apparently our computer 

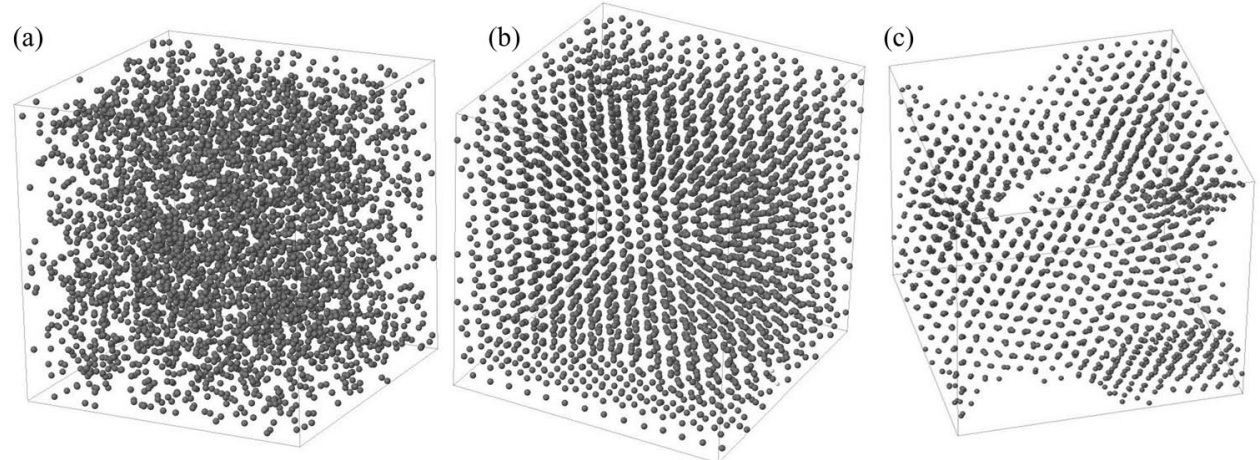

FIG. 2. Snapshots of the configuration during simulations: (a) the particles are initially set randomly in 3D cubic box; (b) the equilibrium configuration with only repulsive Yukawa potential; and (c) the equilibrium configuration with SI potential (with long-range attraction).

simulations offered the evidence in support of the tenability of use of $D_{\text {exp }} / D_{u n i}$ to assess the inhomogeneity of crystal structure.

\section{RESULTS AND DISCUSSIONS}

\section{A. Structure of colloidal crystals}

Our previous study ${ }^{33,34}$ has shown how to use the RS method to identify the types of crystals and also to achieve a series of kinetic parameters during crystallization of charged particles. Typical spectra of bcc and fcc colloidal crystals in our samples are shown in Fig. 3. Due to the limitation of available wave vector $q\left(16-48 \mu \mathrm{m}^{-1}\right.$, calculated from the wavelength of the spectrum) and multiple scattering effects, the volume fraction used here is from 0.006 to 0.02 . The minimum $\Phi$ for crystallization is around 0.002 , but to shorten the crystallization time, we chose $\Phi=0.006$ to be the starting point. From the relative positions of the reflection peaks, we can determine the crystal structures formed by PS1 are bcc and the crystal structures formed by PS4 are fcc for all the investigated $\Phi$.

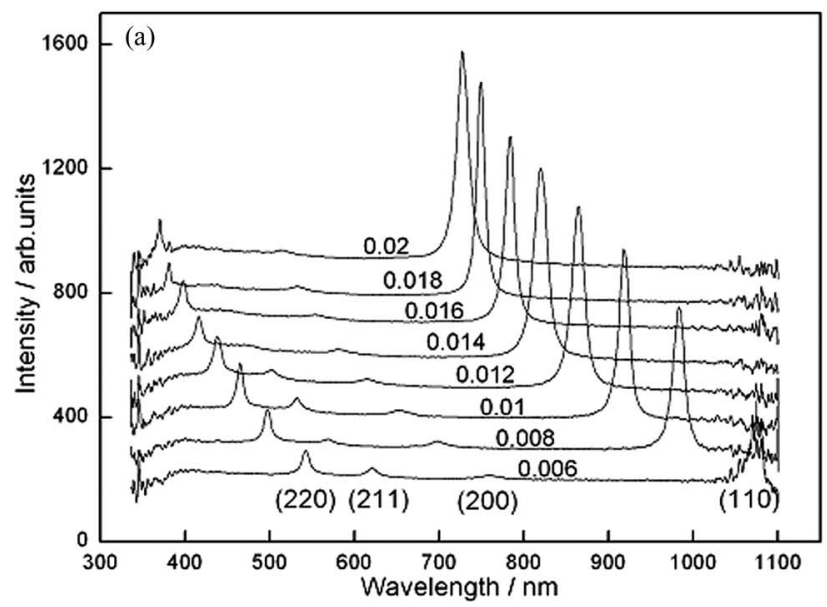

\section{B. Influences of volume fraction and crystal structure to $D_{\text {exp }}$}

The structures and lattice parameters of charged colloidal crystals depend on surface charge and also volume fraction of the particles. Both experiments and computer simulations have shown that low inter-particle repulsion and low volume fraction make colloidal crystals exhibit bcc structure and fcc structure is more favorable for higher repulsion and higher volume fraction. ${ }^{37-40}$

For the five groups of PS particles investigated here, PS1 are bcc in the whole range of $\Phi$ from 0.006 to 0.02 . PS3, PS4, and PS5 which have larger effective charge exhibited fcc structures at the stable state, though bcc may be formed in the early stage of the crystallization process. ${ }^{41}$ However, the crystal formed by middle charged PS2 takes bcc structure when $0.006<\Phi<0.016$ but fcc structure for $\Phi \geq 0.016$. The wavelength of the primary peaks, bcc(110) and fcc(111), for colloidal crystals formed by all the five groups particles are compiled in Table II. From the wavelength of primary peaks, the value of $D_{\text {exp }}$ can be calculated by Eq. (5).

Figure 4(a) shows the curve of $D_{\text {exp }}$ versus $\Phi$ for the group PS2. We can see that $D_{\exp }$ decreases noticeably with

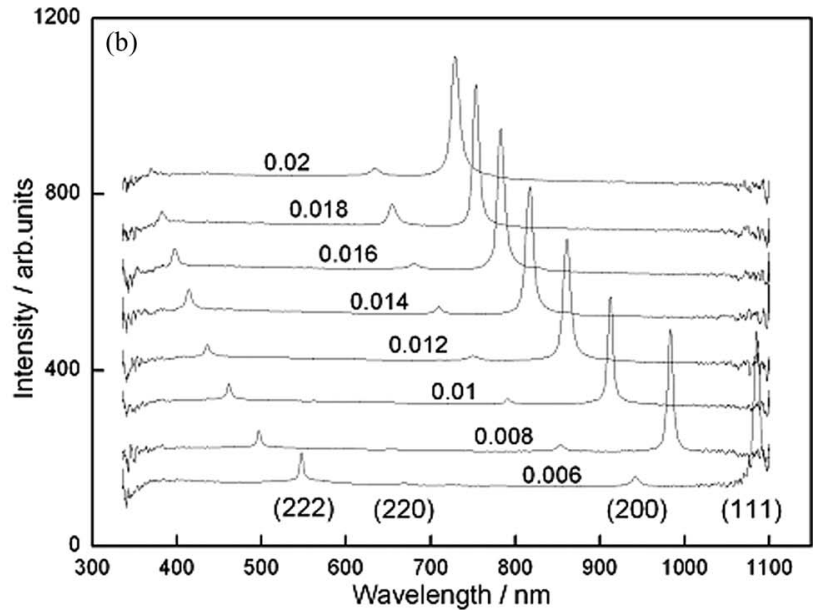

FIG. 3. Reflection spectra of colloidal crystal formed by particles PS1 (a) and PS4 (b) at different $\Phi$. The crystal structures are bcc (PS1) and fcc (PS4), respectively, for all the $\Phi$ from 0.006 to 0.02 . 
TABLE II. Wavelengths of the primary reflection peaks of colloidal crystals formed by all the five groups of particles under different volume fractions.

\begin{tabular}{lrccccccc}
\hline \hline & \multicolumn{7}{c}{ Wavelength (nm) } \\
\cline { 2 - 9 } Sample & 0.006 & 0.008 & 0.010 & 0.012 & 0.014 & 0.016 & 0.018 & 0.020 \\
\hline PS1 & 1084 & 982 & 918 & 865 & 820 & 783 & 749 & 725 \\
PS2 & 909 & 828 & 758 & 712 & 677 & 668 & 640 & 620 \\
PS3 & 946 & 865 & 812 & 765 & 733 & 699 & 676 & 604 \\
PS4 & 1073 & 965 & 900 & 855 & 812 & 773 & 747 & 726 \\
PS5 & 926 & 847 & 787 & 740 & 703 & 673 & 648 & 626 \\
\hline \hline
\end{tabular}

increasing $\Phi$. There is a small jump between $\Phi=0.014$ and $\Phi=0.016$, which is corresponding to a switch of crystal structure from bcc to fcc. In this paper, we are more concerned about the ratio $D_{\text {exp }} / D_{\text {uni }}$ versus $\Phi$, which was shown in Fig. 4(b). Note for a homogeneous bcc or fcc-like structure, the value of $A N N I D$ can be calculated by $D_{u n i}$ $=\sqrt{3} / 2\left(2 / n_{p}\right)^{1 / 3}$ and $D_{u n i}=\sqrt{2} / 2\left(4 / n_{p}\right)^{1 / 3}$, respectively. We found two most striking characteristics: Firstly, though $D_{\text {exp }}$ changed markedly with varying $\Phi, D_{\text {exp }} / D_{u n i}$, an indicator about the crystal is homogeneous or not, basically keeps unchanged. The average value of $D_{\text {exp }} / D_{\text {uni }}$ is about 0.89 , implying the formed crystal is inhomogeneous (voids exist). Secondly, when the suspensions experience a switch from bcc to fcc structures with increasing $\Phi$, no obvious influence to $D_{\text {exp }} / D_{\text {uni }}$ is observed. Again, the inhomogeneous here means the coexistence of voids and ordered structures and in crystal regions the structures are still homogeneous, having equal nearest neighbor inter-particle distance, which is corresponding to $D_{\text {exp }}$ measured by RS.

\section{Surface charge dependence of $D_{\text {exp }} / D_{\text {uni }}$}

More groups of particles with different surface charge were investigated. In Fig. 5(a), the ratio of $D_{\text {exp }} / D_{\text {uni }}$ for all five groups including PS2 are shown. For each particle group, $D_{\text {exp }} / D_{\text {uni }}$ basically keeps unchanged with varying $\Phi$. $D_{\text {exp }} / D_{\text {uni }}$ is about 0.98 for PS1 in the whole range of $\Phi$ from 0.006 to 0.02 , that means the ordered structures fill up the

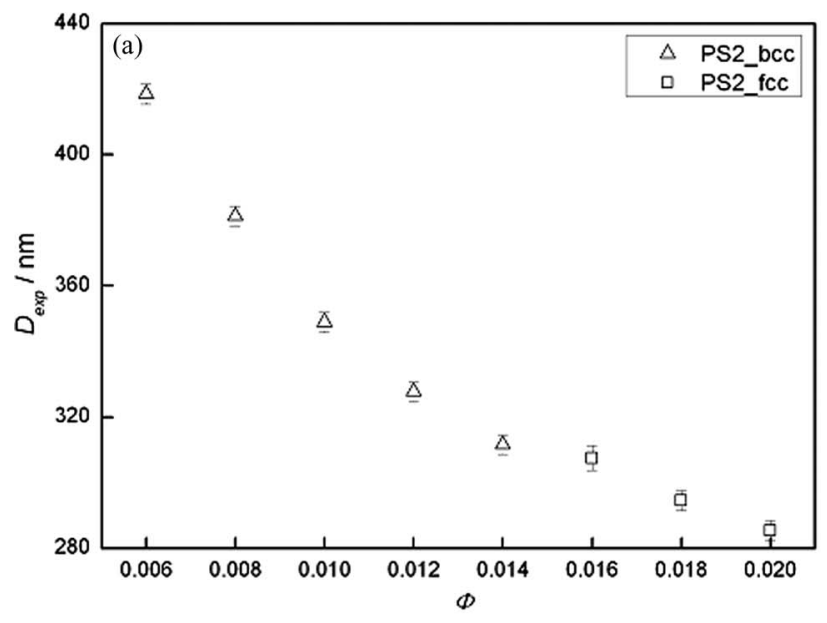

whole space with no voids. We need to take into account that the calculation of $D_{u n i}$ is based on the assumption that only a single crystal is formed, namely, the crystal lattice of the entire sample is continuous and unbroken to the edges of the sample. However, what we obtained in the experiment is actually polycrystal and the defects will make the average interparticle distance be smaller than that for a single crystal. Therefore, it is natural to have $D_{\text {exp }} / D_{\text {uni }}$ equals to 0.98 instead of 1 ; namely, $D_{\text {exp }}$ is $2 \%$ smaller than $D_{\text {uni }}$. We assume that this $2 \%$ of shrinkage is due to the presence of defects in multiple crystals. Now we can see that compared with a single crystal the defects in multiple crystals do cause shrinkage of the average interparticle distance, but this shrinkage is quite small in percentage. For crystals formed by the other groups of particles, the average values of $D_{\text {exp }} / D_{\text {uni }}$ are all smaller than unity markedly (see Fig. 5(a)), but one characteristic is the same, $D_{\text {exp }} / D_{\text {uni }}$ basically keeping unchanged even though the suspension is diluted to twice its original volume. Here we need to stress that the result of $D_{\text {exp }} / D_{\text {uni }}$ remains unchanged probably is true only specially for the range of $\Phi$ in our experiment. Actually, we have seen $D_{\text {exp }} / D_{\text {uni }}$ for PS2 has a bit of tendency to go up toward lower $\Phi(\sim 0.006-0.008)$.

For comparison, the average values of $D_{\text {exp }} / D_{\text {uni }}$ for each group are given in Fig. 5(b). The changing tendency is clear. With the increases of effective charge $Z_{\sigma}^{*}, D_{\text {exp }} / D_{\text {uni }}$ initially decreases quickly and then it approaches a stable value, about 0.85 . The colloidal crystal experiences a homogeneous to inhomogeneous transition, as expected in Ref. 17.

For highly charged colloidal particles PS4 and PS5, their analytical surface charges are 25602 and 18233 , respectively, but their relevant effective charges are only 998 and 1003 (see Table I). This is because the strong coupling of the counterions in the vicinity of the particles makes the effective charge of particles to reach a saturation value, as formulated by the prominent mean-field Poisson-Boltzmann (PB) theory. Quantitatively, Alexander et al. ${ }^{42}$ calculated the relationship between the analytical charge and the effective charge of colloidal particles by solving the PB equation numerically in a spherical Wigner-Seitz (WS) cell. The maximum value of the renormalized effective charge is given by $15 a / \lambda_{B}$, where $\lambda_{B}$ $\left(=e^{2} / \varepsilon_{0} \varepsilon_{r} k_{B} T\right)$ is the Bjerrum length $(\sim 0.72 \mathrm{~nm}), \varepsilon_{0} \varepsilon_{r}$ is the

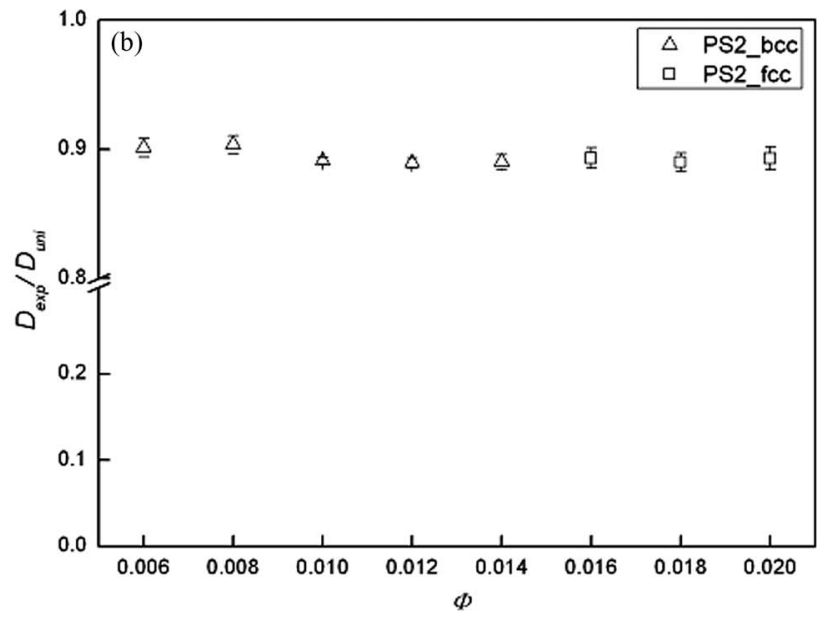

FIG. 4. $\Phi$ dependence of $D_{\exp }$ (a) and $D_{\exp } / D_{\text {uni }}$ (b) for particles PS2. Crystal structure of bcc and fcc are indicated by uptriangle and square, respectively. 

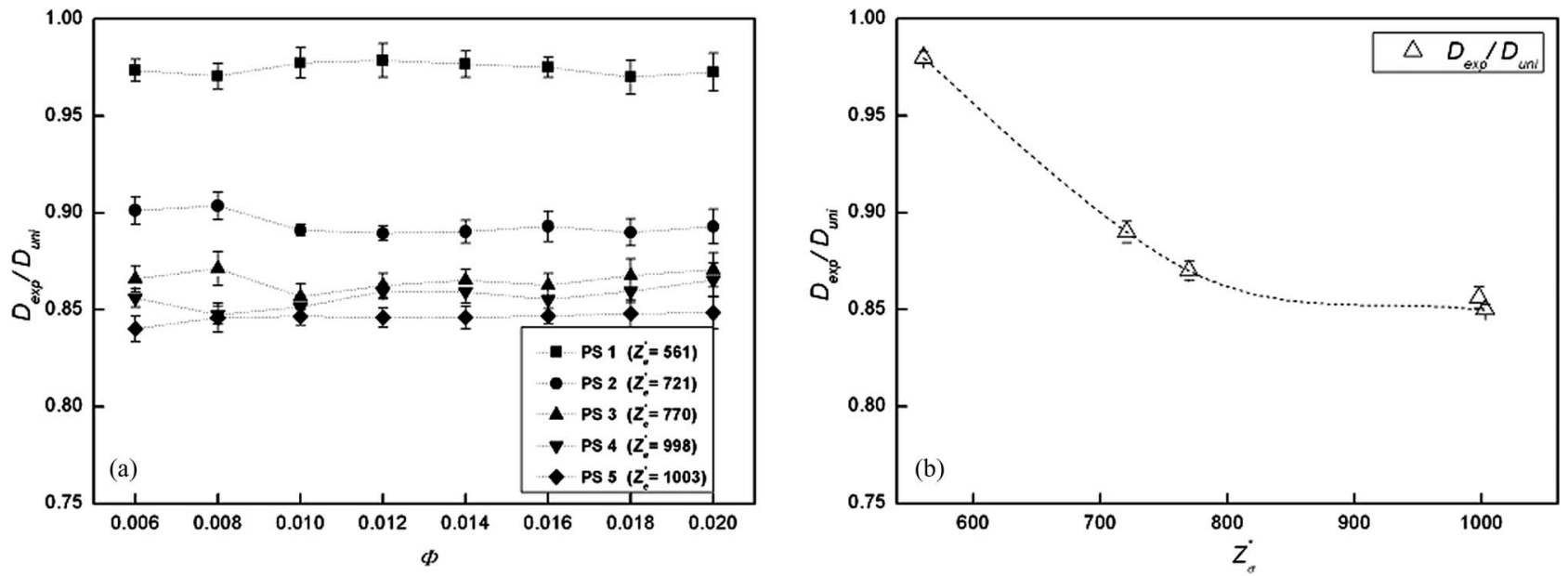

FIG. 5. (a) The ratio of $D_{\text {exp }} / D_{\text {uni }}$ for five groups of particles at different $\Phi$.

(b) Changing tendency of $D_{\text {exp }} / D_{u n i}$ with increasing effective surface charge $Z_{\sigma}^{*}$.

dielectric permittivity of the suspension and $k_{B} T$ is the thermal energy. According to their calculation, the predicated maximum effective charges of PS4 and PS5 are 1208 and 1062, respectively, that are close to our measured values 998 and 1003. This saturation of effective charge suggests that the ratio of $D_{\text {exp }} / D_{u n i}$ has a minimum reachable value. Apparently, in our case this minimum value is around 0.85 .

\section{Comparison between experimental and simulation results}

In order to give explanation to the coexistence of voids and crystallites, some theoretical attempts have been made. The theory of "volume term," proposed by van Roij, appears to succeed in explaining gas-liquid and gas-solid coexistence in the framework of DLVO theory. ${ }^{20,21}$ Sogami and Ise indicated that under certain circumstances, conterions-mediated attractions between like-charged colloidal particles must be taken into consideration; and they further proposed a new form to describe the effective pair-potential $U_{s}(\mathrm{r})$, which includes a long-range attractive term in addition to the usual repulsive screened Coulomb term: ${ }^{18}$

$$
U_{s}(r)=\frac{Z_{e}^{2} e^{2}}{4 \pi \varepsilon_{0} \varepsilon_{r}}\left[\frac{\sinh (\kappa a)}{\kappa a}\right]^{2}\left(\frac{A}{r}-\frac{\kappa}{2}\right) \exp (-\kappa r),
$$

where $A=1+\kappa a \operatorname{coth}(\kappa a), r$ is the closest inter-particle distance, and $a$ is the radius of the particle. $\kappa$ is the inverse Debye-screening length given by ${ }^{43,44}$

$$
\kappa^{2}=\frac{e^{2}}{\varepsilon_{0} \varepsilon_{r} k_{B} T}\left(n Z_{e}+2000 N_{A} C_{\text {salt }}\right),
$$

where $n$ is the particle number density of the suspension $\left(\mathrm{m}^{-3}\right), N_{A}$ is Avogadro's constant, and $C_{\text {salt }}$ is the molar concentration of the foreign salt $\left(\mathrm{mol} \cdot \mathrm{L}^{-1}\right)$. The first term in Eq. (7) represents the counterion contribution and the second term accounts for the excess electrolyte. SI pair-potential has a minimum at $R_{m}$ given by $R_{m}=\left[A+A(A+2)^{1 / 2}\right] / \kappa$ and depth $U_{m}=U_{s}\left(R_{m}\right)$. The structural ordering in the dense phase depends on the magnitude of $U_{m}$. The dense phase is found to have a liquid-like order for $U_{m} \sim k_{B} T$, and a solidlike (crystalline or glass-like) one for $U_{m}>k_{B} T$.
In this paper, for comparison purpose, we also performed MD simulations by using SI potential. As mentioned earlier, our computer simulation only took aim at verification of the study scheme of judging the inhomogeneity of colloidal crystals by means of ANNID measurement. The relevant results presented here, as a typical example, are only for suspension of PS5. More comprehensive results and discussions of the simulation will be presented in a separate publication.

The parameters adopted in the simulations are compatible with those for suspension of PS5. The equilibrium structure is fcc-like crystal for all $\Phi$, which is consistent with experimental result. A comparison between $D_{\text {exp }} / D_{\text {uni }}$ and $D_{\text {sim }} / D_{\text {uni }}$ are listed in Table III. We can see $D_{\text {exp }} / D_{\text {uni }}$ and $D_{s i m} / D_{u n i}$ are in good agreement. The average value of $D_{\text {exp }} / D_{\text {uni }}$ is about 0.85 , and the average value of $D_{\text {sim }} / D_{\text {uni }}$ is about 0.81 . The difference may be come from either effective charge or salt concentration, which is very difficult to be precisely determined. ${ }^{45}$ Note that $D_{\text {sim }} / D_{\text {uni }}<1$, which does indicate the suspension is inhomogeneous, i.e., coexistence of voids and crystals, as revealed in the snapshot of configuration in Fig. 2(c) $(\Phi=0.01)$. On the other hand, all the calculated well depths largely exceed the thermal energy $k_{B} T$, implying the formed crystals are very stable. Our simulation results show that SI potential can satisfactorily explain the experimental phenomena although it does not necessarily mean that SI is the only candidate to account for the experimental observations. Apparently, the most convictive evidence to clarify this problem would be directly measuring the interparticle

TABLE III. A comparison between the results of simulations and experiments.

\begin{tabular}{lllllllll}
\hline \hline & \multicolumn{8}{c}{$\Phi$} \\
\cline { 2 - 7 } & 0.006 & 0.008 & 0.01 & 0.012 & 0.014 & 0.016 & 0.018 & 0.02 \\
\hline$D_{\text {exp }} / D_{\text {uni }}$ & 0.8403 & 0.8459 & 0.8467 & 0.8460 & 0.8461 & 0.8468 & 0.8480 & 0.8485 \\
$D_{\text {sim }} / D_{\text {uni }}{ }^{\mathrm{a}}$ & 0.8114 & 0.8115 & 0.8082 & 0.8097 & 0.8049 & 0.8048 & 0.8081 & 0.8013 \\
$U_{m} / k_{B} T$ & 4.7772 & 5.1492 & 5.4416 & 5.6738 & 5.8589 & 6.006 & 6.1222 & 6.2127 \\
\hline \hline & ${ }^{\text {a }} D_{\text {sim }}$ is the $A N N I D$ of simulation and $D_{\text {exp }}$ is $A N N I D$ of experiment. The diameter of the \\
colloid particle is 102 nm, the effective charge is 1003 , and the estimated salt concentra- \\
tion is $3.0 \times 10^{-6} \mathrm{M}$.
\end{tabular}


interaction experimentally, though such a measurement is very difficult as indicated by Tata. ${ }^{25}$

\section{CONCLUSIONS}

This paper presents an experimental study on the effects of effective surface charge and volume fraction on the homogeneity of the ordered structure by adopting the ratio of $D_{\text {exp }} / D_{\text {uni }}$ to be an indicator. $D_{\text {exp }}$ measurement is achieved by reflection spectroscopy.

The results of our experiments can be summarized into two main points. First, for the particles with the same effective charge, the value of $D_{\text {exp }} / D_{\text {uni }}$ is basically unchanged in the range of $\Phi$ from 0.006 to 0.02 , although $D_{\exp }$ alone drops significantly with increasing $\Phi$. Second, the value of $D_{\text {exp }} / D_{\text {uni }}$ initially drops quickly with increasing effective charge and then tends to an asymptotic value $(\sim 0.85)$. A possible explanation for this "minimum" asymptotic value is due to the saturation of the effective charge. Our preliminary computer simulations also produced results that are consistent with experiments. Our findings appear to conflict with DLVO theory based on the repulsion-only assumption. Inclusion of attraction seems to offer a possible explanation for the existence of voids, although whether this explanation is unique is still worth to further investigate for more profoundly revealing the nature of interaction between charged particles.

\section{ACKNOWLEDGMENTS}

This work is supported by Grant Nos. 11172302, 10972217,11032011 , and 20903112 from the National Natural Science Foundation of China (NNSFC).

${ }^{1}$ W. B. Russel, Nature (London) 421, 490 (2003).

${ }^{2}$ V. J. Anderson and H. N. W. Lekkerkerker, Nature (London) 416, 811 (2002).

${ }^{3}$ A. Yethiraj, Soft Matter 3, 1099 (2007).

${ }^{4}$ Z. R. Wang, F. Wang, Y. Peng, Z. Y. Zheng, and Y. L. Han, Science 338, 87 (2012).

${ }^{5}$ A. Engelbrecht and H. J. Schöpe, Soft Matter 8, 11034 (2012).

${ }^{6}$ B. A. Pailthorpe and W. B. Russel, J. Colloid Interface Sci. 89, 563 (1982).

${ }^{7}$ C. Desgranges and J. Delhommelle, J. Chem. Phys. 126, 054501 (2007).

${ }^{8}$ C. Royall, M. Leunissen, and A. van Blaaderen, J. Phys.: Condens. Matter 15, S3581 (2003).

${ }^{9}$ K. Ito, H. Yoshida, and N. Ise, Science 263, 66 (1994).

${ }^{10}$ P. S. Mohanty, B. V. R. Tata, A. Toyotama, and T. Sawada, Langmuir 21, 11678 (2005).
${ }^{11}$ S. Dosho, N. Ise, K. Ito, S. Iwai, H. Kitano, H. Matsuoka, H. Nakamura, H. Okumura, T. Ono, I. S. Sogami, Y. Ueno, H. Yoshida, and T. Yoshiyama, Langmuir 9, 394 (1993).

${ }^{12}$ N. Ise, T. Konishi, and B. V. R. Tata, Langmuir 15, 4176 (1999).

${ }^{13}$ J. Yamanaka, H. Yoshida, T. Koga, N. Ise, and T. Hashimoto, Phys. Rev. Lett. 80, 5806 (1998).

${ }^{14}$ H. Yoshida, N. Ise, and T. Hashimoto, J. Chem. Phys. 103, 10146 (1995).

${ }^{15}$ H. Yoshida, J. Yamanaka, T. Koga, T. Koga, N. Ise, and T. Hashimoto, Langmuir 15, 2684 (1999).

${ }^{16} \mathrm{H}$. Yoshida, K. Ito, and N. Ise, Phys. Rev. B 44, 435 (1991).

${ }^{17}$ B. V. R. Tata and N. Ise, Phys. Rev. B 54, 6050 (1996).

${ }^{18}$ I. Sogami and N. Ise, J. Chem. Phys. 81, 6320 (1984).

${ }^{19}$ J. T. G. Overbeek, J. Chem. Phys. 87, 4406 (1987).

${ }^{20}$ R. van Roij and J.-P. Hansen, Phys. Rev. Lett. 79, 3082 (1997).

${ }^{21}$ R. van Roij, M. Dijkstra, and J. P. Hansen, Phys. Rev. E 59, 2010 (1999).

${ }^{22}$ K. S. Schmitz and L. B. Bhuiyan, Phys. Rev. E 63, 011503 (2000).

${ }^{23}$ K. S. Schmitz, L. B. Bhuiyan, and A. K. Mukherjee, Langmuir 19, 7160 (2003)

${ }^{24}$ M. V. Smalley, Mol. Phys. 71, 1251 (1990).

${ }^{25}$ J. C. Crocker and D. G. Grier, Phys. Rev. Lett. 77, 1897 (1996); B. V. R. Tata and N. Ise, Phys. Rev. E 58, 2237 (1998); D. G. Grier and J. C. Crocker, ibid. 61, 980 (2000); B. V. R. Tata and N. Ise, ibid. 61, 983 (2000).

${ }^{26}$ W. H. Mulder, Soil Sci. Soc. Am. J. 74, 1 (2010); N. Ise and I. S. Sogami, ibid. 74, 1844 (2010); W. H. Mulder, ibid. 74, 1846 (2010).

${ }^{27}$ G. T. D. Shouldice, G. A. Vandezande, and A. Rudin, Eur. Polym. J. 30, 179 (1994).

${ }^{28}$ K. Ito, N. Ise, and T. Okubo, J. Chem. Phys. 82, 5732 (1985).

${ }^{29}$ D. Hessinger, M. Evers, and T. Palberg, Phys. Rev. E 61, 5493 (2000).

${ }^{30}$ P. Wette, H.-J. Schöpe, R. Biehl, and T. Palberg, J. Chem. Phys. 114, 7556 (2001)

${ }^{31}$ T. Okubo, H. Hase, H. Kimura, and E. Kokufuta, Langmuir 18, 6783 (2002).

${ }^{32}$ M. Murai, T. Okuzono, M. Yamamoto, A. Toyotama, and J. Yamanaka, J. Colloid Interface Sci. 370, 39 (2012).

${ }^{33}$ H. W. Zhou, S. H. Xu, Z. W. Sun, X. Du, and J. C. Xie, Colloid Surf. A 375, 50 (2011).

${ }^{34}$ H. Zhou, S. Xu, Z. Sun, X. Du, and L. Liu, Langmuir 27, 7439 (2011).

${ }^{35}$ D. C. Rapaport, The Art of Molecular Dynamics Simulation (Cambridge University Press, Cambridge, 1997).

${ }^{36}$ H. J. C. Berendsen, J. P. M. Postma, W. F. Vangunsteren, A. Dinola, and J. R. Haak, J. Chem. Phys. 81, 3684 (1984).

${ }^{37}$ Y. Monovoukas and A. P. Gast, J. Colloid Interface Sci. 128, 533 (1989).

${ }^{38}$ H. J. Schöpe, T. Decker, and T. Palberg, J. Chem. Phys. 109, 10068 (1998).

${ }^{39}$ L. Y. Gu, S. H. Xu, Z. W. Sun, and J. T. Wang, J. Colloid Interface Sci. 350, 409 (2010).

${ }^{40}$ M. O. Robbins, K. Kremer, and G. S. Grest, J. Chem. Phys. 88, 3286 (1988).

${ }^{41}$ S. Xu, H. Zhou, Z. Sun, and J. Xie, Phys. Rev. E 82, 010401 (2010).

${ }^{42}$ S. Alexander, P. M. Chaikin, P. Grant, G. J. Morales, P. Pincus, and D. Hone, J. Chem. Phys. 80, 5776 (1984).

${ }^{43}$ A. Engelbrecht, R. Meneses, and H. J. Schöpe, Soft Matter 7, 5685 (2011).

${ }^{44}$ P. Wette, I. Klassen, D. Holland-Moritz, D. M. Herlach, H. J. Schöpe, N. Lorenz, H. Reiber, T. Palberg, and S. V. Roth, J. Chem. Phys. 132, 131102 (2010).

${ }^{45}$ B. V. R. Tata and S. S. Jena, Solid State Commun. 139, 562 (2006). 